\title{
P02-019 - Detection of risk factors for AA-amyloidosis
}

\author{
S Németh ${ }^{1 *}$, L Obici $^{2}$, S Grandemange ${ }^{3}, \mathrm{H}_{\text {Lachmann }}{ }^{4}$, C Oberkanins $^{1}$ \\ From 7th Congress of International Society of Systemic Auto-Inflammatory Diseases (ISSAID) \\ Lausanne, Switerland. 22-26 May 2013
}

\section{Introduction}

Systemic reactive (AA) amyloidosis represents the most important complication within TNF receptor associated periodic syndrome (TRAPS), familial Mediterranean fever (FMF) and other autoinflammatory syndromes, progressively leading to endstage renal failure. The homozygous condition of the serum amyloid A (SAA) variant SAA1.1 is significantly associated with the occurrence of AA amyloidosis in TRAPS patients. Likewise in FMF patients the $M E F V$ mutation c.2080A $>\mathrm{G}$ (M694V) correlates with amyloidosis and the $S A A 1.1 / S A A 1.1$ genotype increases clinical severity (age at disease onset, amyloidosis, arthritis).

\section{Objectives}

To develop a reverse-hybridization assay (Amyloidosis StripAssay) for detection of (1) genetic markers modulating the risk of developing AA amyloidosis in TRAPS and FMF [c.209C >T, c.224T >C (SAA1); c.2080A >G (MEFV)], as well as (2) mannose-binding lectin $2(M B L 2)$ and TNFRSF1A variants, which are suspected to be modulating factors in TRAPS [g.4447 C>G, g.4776 C>G, g.5000 $\mathrm{C}>\mathrm{T}$, c. $154 \mathrm{C}>\mathrm{T}$, c.161G $>\mathrm{A}$, c.170G $>\mathrm{A}(M B L 2)$; c.47333C $>\mathrm{T}($ TNFRSF1A)].

\section{Methods}

The Amyloidosis StripAssay is based on a multiplex PCR and hybridization of biotinylated amplicons under exactly defined stringency to a teststrip presenting a parallel array of allele-specific oligonucleotide probes. Specifically bound PCR products are detected using enzymatic colour reaction.

\section{Results}

The specificity of the StripAssay was validated by hybridizing teststrips against PCR products obtained from plasmid clones, as well as reference DNA samples. All genetic variants covered by the StripAssay could be unambiguously identified.

\section{Conclusion}

The Amyloidosis StripAssay proved to be a fast and reliable method for detection of genetic factors modulating the risk of developing AA amyloidosis in FMF and TRAPS. An association between $M B L 2$ alleles and amyloidosis is not conclusively established, but $M B L 2$ is of considerable diagnostic interest in closely related fields, including rheumatoid arthritis or innate immunity. Also in case of the TNFRSF1A intron 4 polymorphism c. $473-33 \mathrm{C}>\mathrm{T}$ the StripAssay could be a useful tool: the c.473-33C $>\mathrm{T}$ variant is involved in TNFRSF1A expression and an implication on the TRAPS phenotype still needs to be investigated.

\section{Disclosure of interest}

None declared.

\section{Authors' details \\ ${ }^{1}$ ViennaLab Labordiagnostika GmbH, Vienna, Austria. ${ }^{2}$ Biotechnology Research Laboratories, IRCCS Fondazione Policlinico San Matteo, Pavia, Italy. ${ }^{3}$ Unité médicale des maladies auto-inflammatoires, Hôpital Arnaud de Villeneuve, Montpellier, France. ${ }^{4}$ National Amyloidosis Centre, UCL Medical School, London, UK.}

Published: 8 November 2013

doi:10.1186/1546-0096-11-S1-A126

Cite this article as: Németh et al:: P02-019 - Detection of risk factors for AA-amyloidosis. Pediatric Rheumatology 2013 11(Suppl 1):A126.

${ }^{1}$ ViennaLab Labordiagnostika GmbH, Vienna, Austria

Full list of author information is available at the end of the article

(c) 2013 Németh et al; licensee BioMed Central Ltd. This is an Open Access article distributed under the terms of the Creative Commons 